\title{
The X-ray spectral and timing properties of a major radio flare episode in Cygnus X-3
}

\author{
Karri I. I. Koljonen ${ }^{1}$, Diana C. Hannikainen ${ }^{2}$, Michael L. \\ McCollough $^{3}$, Guy G. Pooley ${ }^{4}$, Sergei A. Trushkin ${ }^{5}$, Robert Droulans ${ }^{6}$ \\ ${ }^{1}$ Aalto University Metsähovi Radio Observatory, Metsähovintie 114, 02540 Kylmälä, Finland \\ email: karri@kurp.hut.fi \\ ${ }^{2}$ Department of Physics and Space Sciences, Florida Institute of Technology, $150 \mathrm{~W}$. \\ University Blvd., Melbourne, FL 32901, USA \\ ${ }^{3}$ Smithsonian Astrophysical Observatory, 60 Garden Street, Cambridge, MA 02138, USA \\ ${ }^{4}$ Astrophysics Group, Cavendish Laboratory, 19 J. J. Thomson Avenue, Cambridge, UK \\ ${ }^{5}$ Special Astrophysical Observarory RAS, Nizhnij Arkhyz, 36916, Russia \\ ${ }^{6}$ CNRS; IRAP; 9 Av. colonel Roche, BP 44346, F-31028 Toulouse cedex 4, France
}

\begin{abstract}
The microquasar Cygnus X-3 is known for massive outbursts that emit radiation from radio to $\gamma$-rays associated with jet ejection events. Using Principal Component Analysis to probe fast $(\sim 1 \mathrm{~min}) \mathrm{X}$-ray spectral evolution followed by subsequent spectral fits to the timeaveraged spectra $(\sim 3 \mathrm{ks})$, we find that the overall X-ray variability during major outbursts can be attributed to two components. The spectral evolution of these components are best fitted with hybrid Comptonization and thermal bremsstrahlung components. Most of the X-ray variability is attributed to the hybrid Comptonization component. However, the spectral evolution of the thermal component is linked to a change in the X-ray spectral state. Phase-folding the fit results shows that the thermal component is restricted to two orbital phase regions opposite to each other, possibly indicating a flattened stellar wind from the Wolf-Rayet companion.
\end{abstract}

Keywords. Accretion, accretion disks - Binaries: close - Stars: winds, outflows - X-rays: binaries - X-rays: individual: Cygnus X-3

\section{Observations and Analysis}

We applied principal component analysis (PCA) to sets of X-ray spectra from a 2006 May-June multiwavelength campaign (see Fig. 1) following the procedure presented in Malzac et al. 2006. PCA is used here as a variability analysis tool which allows one to combine temporal and spectral information to identify various components of the spectrum based on their variability. The main use of PCA is to find the least amount of components that are enough to explain the data losing the least amount of information in the process. By finding the principal components of the data set which explain most of the observed variability the dimensionality of the dataset is reduced. Fig. 2 shows an example variance spectrum using $R X T E$ data. The variance spectra from Swift and INTEGRAL produced similar results. Based on PCA we find that

- The variability of the X-ray spectra of Cyg X-3 during major flare ejections are produced mainly by two components.

- The first and second principal components $\left(\alpha_{1}, \alpha_{2}\right)$ show a linear relationship between the two components.

- A change between intermediate spectral states from harder to softer states occurs with increasing values of $\alpha_{2}$.

- The variability occurs in bimodal fashion in orbital phase for both components. 


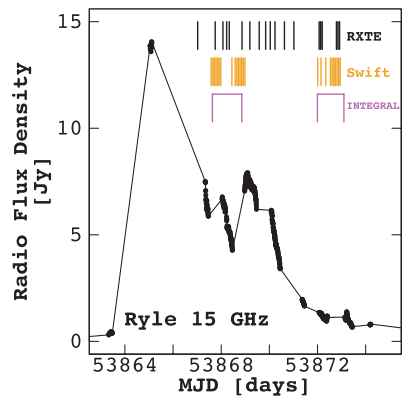

Figure 1. RXTE, Swift and INTEGRAL observations taken during the 2006 May-June major radio flare episode (one outburst shown here). Supporting radio data are from observatories Ryle/AMI and RATAN-600.

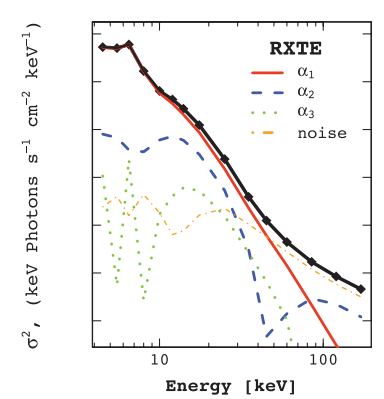

Figure 2. $R X T E$ variance spectrum. Most of the variance $(97 \%)$ is caused by the first principal component $\left(\alpha_{1}\right)$. The second component $\left(\alpha_{2}\right)$ contributes $2.6 \%$, third $\left(\alpha_{3}\right) \quad 0.3 \%$ and the rest together $0.1 \%$ labeled as noise.

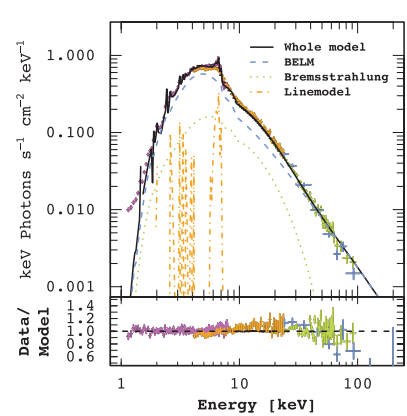

Figure 3. An example of the energy spectrum during the major radio flare episode from simultaneous RXTE, Swift and INTEGRAL observations (see Fig. 1) with best-fit model (components labeled).

\section{Spectral Modeling}

PCA sets a second requirement for the X-ray spectral fits: they have to satisfy also the spectral evolution inferred from the PCA, reducing the number of suitable models. The X-ray spectra of Cyg X-3 are well fitted by hybrid Comptonization (Koljonen et al. 2010) and we assume that $\alpha_{1}$ is coupled to this component. However, we find that most of the spectra studied are not well fitted using just Comptonization. Also, based on PCA, $\alpha_{2}$ is needed in order to explain all the X-ray variability. Out of multiple different models the only model that causes the necessary variability of the principal components is hybrid Comptonization (BELM, Belmont et al. 2008) with thermal bremsstrahlung (see Fig. 3).

\section{Origin of the thermal component}

The most plausible origin of the thermal component is a plasma cloud that forms as a result of the compact object colliding with the WR stellar wind (Zdziarski et al. 2010). Thermal bremsstrahlung emission becomes a substantial source for photons which get upscattered by Comptonization in the cloud. The orbital changes in the bremsstrahlung normalization $\left(\sim n_{i} n_{e}\right)$, which correlates with $\alpha_{2}$, could arise if the wind is asymmetric.

Thermal, hot components have also been found in GRS 1915+105 (Titarchuk \& Seifina 2009, Mineo et al. 2012), SS 433 (Seifina \& Titarchuk 2012) and several XRBs (Zýcki et al. 2001), thus raising questions if this is something intrinsic to microquasars/XRBs, if the emission mechanism is the same and if this scenario could be extended to disk winds?

\section{References}

Belmont, R., Malzac, J., \& Marcowith, A. 2008, A\&A, 491, 617

Koljonen, K. I. I. et al. 2010, MNRAS, 406, 307

Malzac, J. et al. 2006, A\&SA, 448, 1125

Mineo, T. et al. 2012, A\&A $, 537,18$

Seifina, E. \& Titarchuk, L. 2010, ApJ, 722, 586

Titarchuk, L. \& Seifina, E. 2009, ApJ, 706, 1463

Zdziarski, A. A., Misra, R., \& Gierlinski, M. 2010, MNRAS, 402, 767

Zẏcki, P. T., Done, C., \& Smith, D. A. 2001, MNRAS, 326, 1367 